\title{
The influence of tertiary aromatic amines on the BPO initiated cure of unsaturated epoxy polyesters with styrene studied by non-isothermal DSC
}

\author{
Marta Worzakowska
}

Received: 23 October 2010/Accepted: 5 April 2011/Published online: 28 April 2011

(C) The Author(s) 2011. This article is published with open access at Springerlink.com

\begin{abstract}
The influence of tertiary aromatic amines on the course of BPO initiated cure reaction of unsaturated epoxy polyesters with different styrene content has been studied by non-isothermal differential scanning calorimetry. Unsaturated epoxy polyesters prepared from cyclohex-4ene-1,2-dicarboxylic anhydride, maleic anhydride and suitable aliphatic glycol: ethylene glycol or 1,4-butanediol or 1,6-hexanediol were dissolved in vinyl monomer (styrene) resulting in a styrene content of $20-80 \%$ by weight. The styrene solutions of polyesters were subjected to the cure reaction with suitable curing agent: benzoyl peroxide (BPO) used in various concentration $(0.5-3.0 \mathrm{wt} \%)$ or the mixture of $\mathrm{BPO} /$ stoichiometric ratio of chosen tertiary aromatic amines: ( $N, N$-dimethylbenzylamine (BDMA) or 2,4,6-tri(dimethylaminomethyl) phenol (DMP-30). The curing characteristic such as: temperature of the cure initiation $\left(T_{\text {onset }}\right)$, peak maximum temperature $\left(T_{\max }\right)$, final cure temperature $\left(T_{\text {end }}\right)$, heat generated during the cure reaction $(\Delta H)$ were evaluated. It has been found that the course of the cure reaction depended on the styrene content in prepared compositions and the initiating system used. The performed investigations confirmed that one of the applied tertiary aromatic amine: BDMA was an effective promoter for BPO decomposition process, causing a decrease in characteristic curing temperatures of unsaturated epoxy polyesters with
\end{abstract}

M. Worzakowska ( $\square)$

Department of Polymer Chemistry, Faculty of Chemistry,

Maria Curie Sklodowska University, Gliniana 33 Street,

20-614 Lublin, Poland

e-mail: marta.worzakowska@ poczta.umcs.lublin.pl styrene. The organic peroxide-amine interactions caused the promotion of BPO decomposition to benzoyloxy radicals at lower temperatures and thus accelerated the copolymerization process. However, DMP-30 was a very sluggish promoter for BPO decomposition, probably due to the presence of both hydroxyl group, their ortho-position to two of three amine groups and their branched structure. The redox reaction between BPO and DMP-30 probably resulted in non-radical products or radical formation which was incapable of initiating the polymerization reaction.

Keywords Unsaturated epoxy polyester - DSC - Tertiary aromatic amines $\cdot$ Benzoyl peroxide $\cdot$ Cure reactions

\section{Introduction}

In general, the cure of thermosets such as unsaturated polyester and epoxy resins, proceeds by transformation from liquid to rubber (gel) to glass to form a rigid cross-linked structure [1,2]. The cure reaction of unsaturated polyester resins is a free radical chain-growth copolymerization between carbon-carbon double bonds of vinyl monomer, e.g., styrene, divinylbenzene, methyl (meth)acrylate, and unsaturated double bonds in polyester's chain [3]. In general, to induce this cure reaction, various types of organic peroxides, e.g., ketone or alkyl hydroperoxides, diacyl or dialkyl peroxides, which decompose to form active species at elevated temperature or with the addition of promoters are commonly used $[4,5]$.

In contrast, epoxy resins can be cured with a number of nucleophilic or electrophilic reagents, e.g., polycarboxylic acid anhydrides, imidazoles, or amines [6-8]. The various type of amines, both mono- or polyamines, e.g., $\mathrm{N}, \mathrm{N}$ dimethylaniline (DMA); $N, N$-dimethyl-p-toluidine (DMPT); 
diaminodiphenyl methane (DDM) could be used as a curing agents of epoxy groups or as catalysts of the cure reaction of epoxy groups with acid anhydrides resulting in totally different molecular architecture of cured products: fully crosslinked structure (with polyamines) or a linear structure (with monoamines) [9, 10].

In the cure of epoxy using tertiary amine curing agent, the initial step of polymerization is the formation of a tertiary amine-epoxide zwitterion (the negatively charged oxygen) which attacks another epoxy ring to form an oligomer (polymer) via an anionic step growth mechanism [10]. As it is generally known, the epoxy-amine reaction is catalyzed by the presence of hydroxyl groups in the reagent's structure, or introduced with other compounds, e.g., water, alcohol, or moisture. This allowed initiation and acceleration of the epoxy-amine cure process due to the formation of hydrogen-bonding groups [11, 12]. Moreover, based on literature data, tertiary aromatic amines are most commonly applied as promoters for the cure reaction of unsaturated polyester resins initiated by organic peroxides. This applied "catalytic" system consisting of organic peroxide and tertiary aromatic amine makes possible free radical formation at temperatures below those required for the peroxide alone [13-15]. The mechanism of decomposition of BPO by tertiary aromatic amines (e.g., DMA, DMPT, and N,N-dimethyl-m-toluidyne (DDMT) in non-aqueous media has been already studied. The promotion of BPO by those tertiary aromatic amines depend on the formation of the complex ( $N$-benzoyloxydimethylanilinium ion) which decomposes to radical cations of tertiary amine and a benzoyloxy radical, which initiates the cure reaction of unsaturated polyesters with vinyl monomer [9, 16].

This article describes studies of selected tertiary aromatic amines as potential promoters of the cure of unsaturated epoxy polyester resins with styrene initiated benzoyl peroxide. The experiments were performed at a heating rate of $10 \mathrm{~K} \mathrm{~min}^{-1}$ in the temperature range of $20-500{ }^{\circ} \mathrm{C}$ under nitrogen atmosphere. The temperature of the cure initiation $\left(T_{\text {onset }}\right)$, peak maximum temperature $\left(T_{\max }\right)$, final cure temperature $\left(T_{\text {end }}\right)$, heat generated during the cure reaction $(\Delta H)$ as well as the thermal degradation temperature $\left(T_{\mathrm{d}}\right)$ were evaluated.

\section{Experimental}

\section{Materials}

Unsaturated epoxy polyesters (UEP) were prepared by chemical modification of unsaturated polyesters based on cyclohex-4-ene-1,2-dicarboxylic anhydride, maleic anhydride (MA), Merck-Schuchardt (Germany), and suitable glycol: ethylene glycol (EG) or 1,4-butanediol (BDO) or 1,6-hexanediol (HDO), Fluka, Chemika (Switzerland) according to procedure described in [17]. Styrene was delivered by Polish Chemical Reagents-POCH. Benzoyl peroxide (BPO) (98\% purity) was purchased from MerckSchuchardt (Germany). BDMA and DMP-30 were produced by Ted Pella, Inc., CA (USA). All reagents were used as received. The structure of applied radical initiator and tertiary aromatic amines was shown in Scheme 1.

\section{Curing procedure}

The styrene solutions of UEP based on suitable aliphatic glycol: EG, BDO, or HDO containing different styrene content (20-80 wt\%) were prepared. Benzoyl peroxide at concentrations of $0.5-3.0 \mathrm{wt} \%$ was used as a radical initiator. Also, stoichiometric ratio of suitable tertiary aromatic amine: BDMA or DMP-30 in the mixture with BPO was applied as promoters of BPO decomposition as well as standalone hardener of epoxy groups. The experimental samples were prepared by mixing suitable amount of styrene with BPO and/or promoter and added to unsaturated epoxy polyesters, mixed to obtain homogeneous solutions. The prepared compositions were tested immediately after mixing by non-isothermal differential scanning calorimetry (DSC).

\section{Techniques}

The calorimetric measurements were carried out in the Netzsch DSC 204 calorimeter (Germany). The dynamic scans were performed at a heating rate of $10 \mathrm{~K} \mathrm{~min}^{-1}$ from room temperature to a maximum of $500{ }^{\circ} \mathrm{C}$ under nitrogen atmosphere $\left(30 \mathrm{~mL} \mathrm{~min}{ }^{-1}\right)$. As a reference an empty aluminum crucible was used.
Scheme 1 The structure of radical initiator and tertiary aromatic amines used

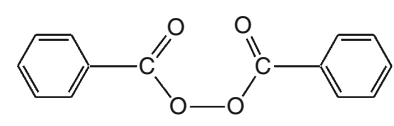

BPO

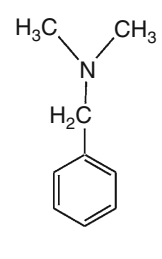

BDMA<smiles>CN(C)Cc1cc(CN(C)C)c(O)c(CN(C)C)c1</smiles>

DMP-30 


\section{Results and discussion}

The cure behavior of the unsaturated epoxy polyester systems was studied by non-isothermal DSC analysis. The tertiary aromatic amines were used as potential promoters of BPO decomposition as well as hardener of epoxy groups. As a baseline for comparison, the cure of systems initiated with only BPO (no amine) as well as samples containing only amine (no BPO) were examined. The samples were heated from 20 to $500{ }^{\circ} \mathrm{C}$, in order to evaluate thermal effects due to cross-linking and decomposition of the cured material under nitrogen. The curing characteristics such as: temperature of the cure initiation $\left(T_{\text {onset }}\right)$, peak maximum temperature $\left(T_{\max }\right)$, final cure temperature $\left(T_{\text {end }}\right)$, the heat generated during the cure reaction $(\Delta H)$ obtained by the integration of the thermal peaks, and also the decomposition temperature $\left(T_{\mathrm{d}}\right)$ were evaluated. Tables 1 and 2 contain the DSC data of the cure reaction of unsaturated epoxy polyesters based on EG, BDO, or HDO with different styrene contents as well as various BPO concentrations, respectively. DSC curves for these samples are shown in Figs. 1 and 2. In general, BPO initiated cure reaction of the studied polyesters with styrene was described by two asymmetric, exothermal peaks which were related to free radical chain-growth copolymerization and/or styrene homopolymerization $\left(T_{\max 1}\right)$ and thermal curing of epoxy groups $\left(T_{\max 2}\right)$, as was proved in previous publication [18]. Moreover, it is apparent from the scanning DSC results, that $T_{\text {onset }}$ values decrease but $T_{\max 1}$ and $T_{\text {end1 }}$ values rise with increasing styrene content, see Table 1. The differences in $T_{\text {onset }}$ values are probably associated with the oxygen concentration dissolved in the resin during sample preparation (from air) and consequently with the viscosity of prepared compositions. It is

Table 1 DSC data of the cure reaction of unsaturated epoxy polyesters based on EG, BDO, or HDO: different styrene content (20-80 wt $\%)$ initiated by BPO (1.0 wt\%)

\begin{tabular}{|c|c|c|c|c|c|c|c|}
\hline Polyester & Styrene content/wt $\%$ & $T_{\text {onset }} /{ }^{\circ} \mathrm{C}$ & $T_{\max 1} /{ }^{\circ} \mathrm{C}$ & $T_{\max 2} /{ }^{\circ} \mathrm{C}$ & $T_{\text {end } 1} /{ }^{\circ} \mathrm{C}$ & $\Delta H_{1} / \mathrm{J} \mathrm{g}^{-1}$ & $T_{\mathrm{d}} /{ }^{\circ} \mathrm{C}$ \\
\hline \multirow[t]{4}{*}{ EG } & 20 & 85 & 121 & 310 & 154 & 117 & 366 \\
\hline & 40 & 80 & 123 & 316 & 159 & 153 & 382 \\
\hline & 60 & 76 & 125 & 318 & 163 & 171 & 410 \\
\hline & 80 & 73 & 130 & 320 & 165 & 188 & 418 \\
\hline \multirow[t]{4}{*}{ BDO } & 20 & 86 & 122 & 328 & 155 & 118 & 373 \\
\hline & 40 & 78 & 124 & 334 & 160 & 152 & 395 \\
\hline & 60 & 75 & 128 & 333 & 165 & 174 & 410 \\
\hline & 80 & 70 & 132 & 335 & 166 & 190 & 420 \\
\hline \multirow[t]{4}{*}{ HDO } & 20 & 88 & 127 & 331 & 150 & 114 & 406 \\
\hline & 40 & 80 & 129 & 335 & 156 & 150 & 408 \\
\hline & 60 & 77 & 132 & 337 & 164 & 173 & 412 \\
\hline & 80 & 75 & 137 & 338 & 168 & 187 & 419 \\
\hline
\end{tabular}

Table 2 DSC data of the cure reaction of unsaturated epoxy polyesters based on EG, BDO, or HDO: styrene (20 wt $\%)$ initiated by different BPO concentration $(0.5-3.0 \mathrm{wt} \%)$

\begin{tabular}{llllllrl}
\hline Polyester & $\mathrm{BPO}$ concentration/wt $\%$ & $T_{\text {onset }}{ }^{\circ} \mathrm{C}$ & $T_{\max } 1^{\circ} \mathrm{C}$ & $T_{\max 2} /{ }^{\circ} \mathrm{C}$ & $T_{\text {end } 1} /{ }^{\circ} \mathrm{C}$ & $\Delta H_{1} / \mathrm{J} \mathrm{g}^{-1}$ & $T_{\mathrm{d}}{ }^{\circ} \mathrm{C}$ \\
\hline EG & 0.5 & 95 & 129 & 310 & 170 & 97 & 366 \\
& 1.0 & 85 & 121 & 310 & 154 & 117 & 366 \\
& 2.0 & 78 & 110 & 312 & 150 & 148 & 368 \\
& 3.0 & 75 & 107 & 311 & 145 & 176 & 370 \\
BDO & 0.5 & 98 & 134 & 325 & 190 & 98 & 373 \\
& 1.0 & 86 & 122 & 328 & 155 & 118 & 373 \\
& 2.0 & 80 & 109 & 328 & 150 & 148 & 375 \\
& 3.0 & 78 & 105 & 330 & 140 & 178 & 380 \\
HDO & 0.5 & 100 & 139 & 330 & 186 & 95 & 405 \\
& 1.0 & 88 & 127 & 331 & 150 & 114 & 406 \\
& 2.0 & 85 & 109 & 331 & 155 & 150 & 404 \\
& 3.0 & 80 & 102 & 332 & 151 & 177 & 402 \\
\hline
\end{tabular}




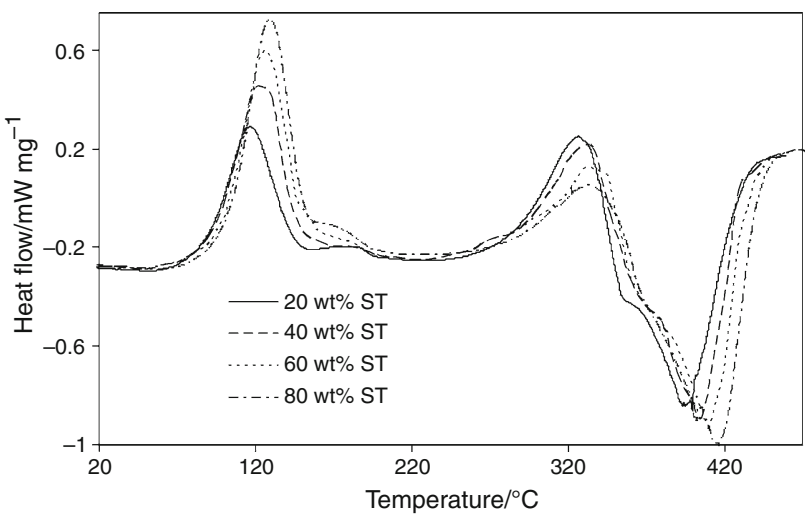

Fig. 1 DSC curves of the cure reaction of unsaturated epoxy polyester based on BDO-different styrene content (20-80 wt\% ST) initiated by $1.0 \mathrm{wt} \%$ of BPO

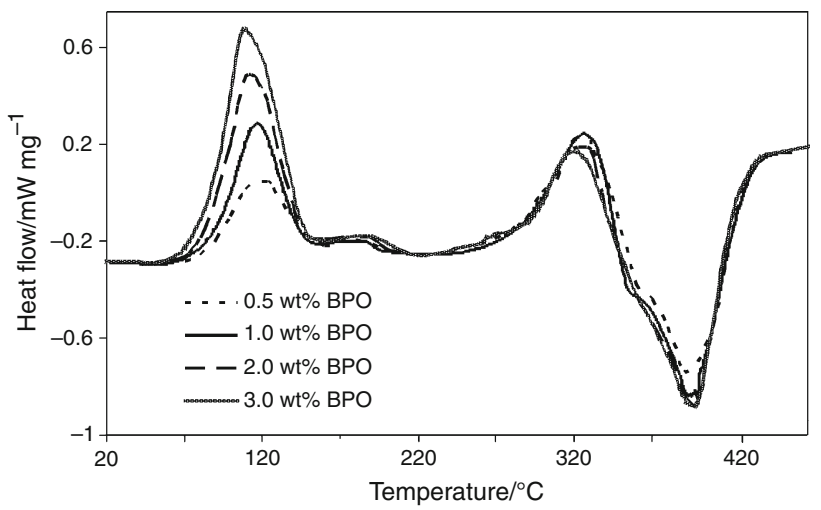

Fig. 2 DSC curves of the cure reaction of unsaturated epoxy polyester based on BDO-styrene (20 wt \% ST) initiated by different BPO concentration

well known that oxygen is an efficient inhibitor of conventional radical polymerization [19]. Probably due to lower viscosity of high styrene content samples, faster oxygen diffusion from the resin in DSC chamber causes a decrease in the induction period of the cure process and thus lower $T_{\text {onset }}[20,21]$. Moreover, it is reasonable to expect a constant consumption rate of both monomers at lower styrene content in the composition. However, mostly polystyrene formation is expected initially but later in the reaction styrene-polyester copolymerization is expected due to higher molecular mobility of styrene molecules at higher styrene content in a lower cross-linked state. Nevertheless, when homopolymerization of styrene is achieved, the copolymerization remains still active and thus after this curing period, the formation of copolymer is expected [22]. Probably, due to the steric hindrance, the movement of reacting groups was significantly restricted for higher styrene content compositions and thus resulted in increasing of $T_{\max 1}$ and $T_{\text {end1 } 1}$ temperatures. Also, increasing of the heat of polymerization $\left(\Delta H_{1}\right)$ with increasing styrene content is directly visible. This observation was mainly connected with styrene (their homopolymerization), which was confirmed by DSC analysis. The BPO initiated (1.0 wt $\%)$ cure process of pure styrene was described by one exothermal effect with following values: $T_{\text {onset }}=75{ }^{\circ} \mathrm{C}, T_{\max }=136{ }^{\circ} \mathrm{C}, T_{\text {end }}=$ $210{ }^{\circ} \mathrm{C}$, and $\Delta H=190 \mathrm{Jg}^{-1}$ in the studied experimental conditions.

The effect of BPO concentration on the course of the cure reaction was determined for the resins containing various concentrations of radical initiator. The cure process of unsaturated epoxy polyesters was described by two, exothermal effects, Figs. 1 and 2. Moreover, $T_{\text {onset }}$ and $T_{\max 1}$ values decreased with increasing concentration of BPO, Table 2. This observation was in accordance with literature data, where for ideal free radical chain-growth polymerization, the rate of inhibitor consumption as well as the rate of copolymerization reaction increased with higher initiator concentration [19]. The total heat of polymerization is the sum of the heat of copolymerization and/or homopolymerization and the heat of decomposition of organic peroxide [23, 24]. The decomposition of BPO, as was confirmed in previous article [18], was highly exothermal reaction so BPO concentration significantly influenced on $\Delta H_{1}$ values. It is clearly seen that higher $\mathrm{BPO}$ concentration in the studied compositions is given higher values of $\Delta H_{1}$. However, the position of the second exotherm temperature peak $\left(T_{\max 2}\right)$ was independent of BPO concentration. It definitely confirmed that BPO concentration had no influence on the course of the thermal curing of epoxy groups.

Tables 3 and 4 contain the DSC data of the cure reaction of unsaturated epoxy polyesters with different styrene contents as well as various BPO concentrations/stoichiometric ratio of BDMA, respectively. DSC curves for these samples are shown in Figs. 3 and 4. BPO/BDMA initiated cure reaction of the studied polyesters with styrene was described by one broad non-entirely divided exothermal peak with two maxima $\left(T_{\max 1}\right.$ and $\left.T_{\max 2}\right)$. The cure process started at relatively lower $T_{\text {onset }}$ temperatures compared to those only initiated by BPO. It could be confirmed that the presence of tertiary aromatic amine: BDMA might accelerate of the cure reaction and promote the copolymerization process due to the chemical interactions between organic peroxide-amine, which lead to the production of benzoyloxy radicals at relatively lower temperature [16]. Moreover, $T_{\text {onset }}$ values decrease but $T_{\max 1}$ and $T_{\text {end }}$ values of BPO/BDMA initiated cure process rise with increasing styrene content, see Table 3 . Also, $T_{\text {onset }}$ and $T_{\max 1}$ values decrease with increasing $\mathrm{BPO}$ concentration for $\mathrm{BPO} /$ BDMA initiating system, Table 4 . The results were comparable to those obtained for BPO initiated reaction and were discussed above. The second peak $\left(T_{\max 2}\right)$ occurs at similar temperatures for BPO/BDMA and only BDMA 
Table 3 DSC data of the cure reaction of unsaturated epoxy polyesters based on EG, BDO, or HDO: different styrene content (20-80 wt\%) initiated by BPO $(1.0 \mathrm{wt} \%) / \mathrm{BDMA}$

\begin{tabular}{|c|c|c|c|c|c|c|c|}
\hline Polyester & Styrene content $/ \mathrm{wt} \%$ & $T_{\text {onset }}{ }^{\circ} \mathrm{C}$ & $T_{\max 1} /{ }^{\circ} \mathrm{C}$ & $T_{\max 2} /{ }^{\circ} \mathrm{C}$ & $T_{\text {end }} /{ }^{\circ} \mathrm{C}$ & $\Delta H_{1}+\Delta H_{2} / \mathrm{J} \mathrm{g}^{-1}$ & $T_{\mathrm{d}} /{ }^{\circ} \mathrm{C}$ \\
\hline \multirow[t]{4}{*}{ EG } & 20 & 65 & 124 & 170 & 254 & 277 & 380 \\
\hline & 40 & 60 & 125 & 172 & 259 & 308 & 385 \\
\hline & 60 & 58 & 128 & 174 & 263 & 323 & 395 \\
\hline & 80 & 50 & 135 & 170 & 265 & 348 & 410 \\
\hline \multirow[t]{4}{*}{ BDO } & 20 & 64 & 126 & 182 & 255 & 283 & 385 \\
\hline & 40 & 59 & 124 & 181 & 260 & 312 & 390 \\
\hline & 60 & 60 & 129 & 180 & 265 & 333 & 400 \\
\hline & 80 & 53 & 135 & 185 & 266 & 352 & 415 \\
\hline \multirow[t]{4}{*}{$\mathrm{HDO}$} & 20 & 70 & 128 & 185 & 250 & 279 & 395 \\
\hline & 40 & 65 & 133 & 187 & 256 & 320 & 400 \\
\hline & 60 & 58 & 138 & 190 & 264 & 339 & 410 \\
\hline & 80 & 55 & 139 & 192 & 268 & 342 & 418 \\
\hline
\end{tabular}

Table 4 DSC data of the cure reaction of unsaturated epoxy polyesters based on EG, BDO, or HDO: styrene (20 wt $\%)$ initiated by different BPO concentration (0.5-3.0 wt \%)/BDMA

\begin{tabular}{|c|c|c|c|c|c|c|c|}
\hline Polyester & BPO concentration/wt $\%$ & $T_{\text {onset }} /{ }^{\circ} \mathrm{C}$ & $T_{\max 1} /{ }^{\circ} \mathrm{C}$ & $T_{\max 2} /{ }^{\circ} \mathrm{C}$ & $T_{\text {end }} /{ }^{\circ} \mathrm{C}$ & $\Delta H_{1}+\Delta H_{2} / \mathrm{J} \mathrm{g}^{-1}$ & $T_{\mathrm{d}}{ }^{\circ} \mathrm{C}$ \\
\hline \multirow[t]{4}{*}{ EG } & 0.5 & 80 & 133 & 170 & 255 & 247 & 382 \\
\hline & 1.0 & 60 & 124 & 170 & 254 & 277 & 380 \\
\hline & 2.0 & 55 & 115 & 175 & 250 & 290 & 381 \\
\hline & 3.0 & 53 & 109 & 176 & 254 & 312 & 385 \\
\hline \multirow[t]{4}{*}{$\mathrm{BDO}$} & 0.5 & 85 & 136 & 180 & 255 & 250 & 386 \\
\hline & 1.0 & 59 & 126 & 182 & 255 & 283 & 385 \\
\hline & 2.0 & 65 & 112 & 185 & 260 & 295 & 385 \\
\hline & 3.0 & 58 & 110 & 187 & 260 & 315 & 390 \\
\hline \multirow[t]{4}{*}{ HDO } & 0.5 & 96 & 142 & 185 & 255 & 243 & 395 \\
\hline & 1.0 & 70 & 128 & 185 & 250 & 279 & 395 \\
\hline & 2.0 & 65 & 111 & 190 & 260 & 290 & 397 \\
\hline & 3.0 & 60 & 106 & 182 & 258 & 310 & 392 \\
\hline
\end{tabular}

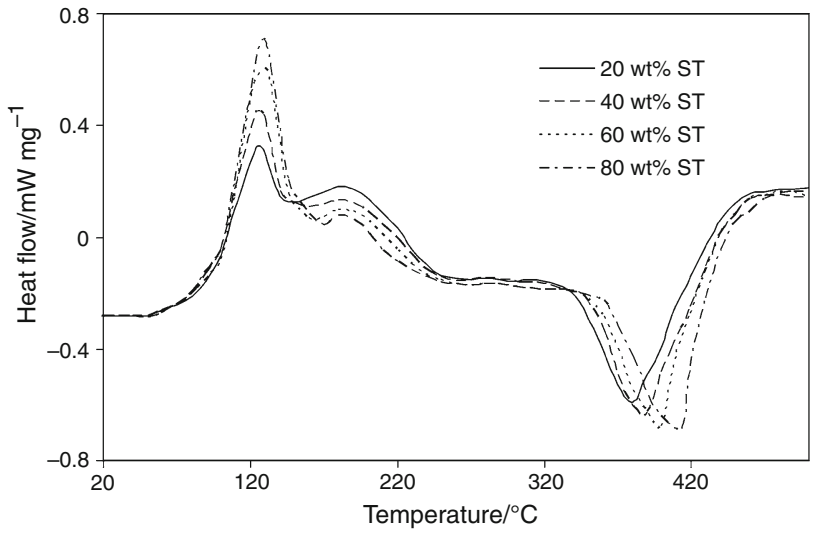

Fig. 3 DSC curves of the cure reaction of unsaturated epoxy polyester based on BDO-different styrene content (20-80 wt $\%$ ST) initiated by $1.0 \mathrm{wt} \%$ BPO/BDMA system

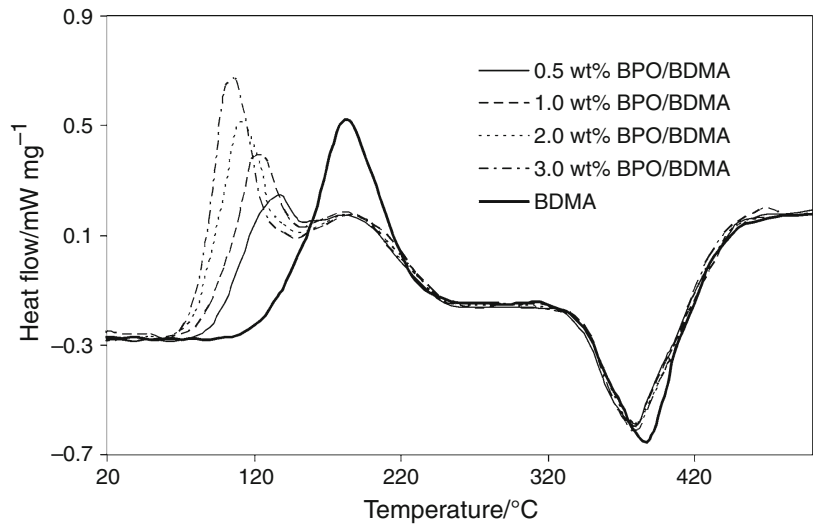

Fig. 4 DSC curves of the cure reaction of unsaturated epoxy polyester based on BDO-styrene (20 wt $\%$ ST) initiated by different BPO concentration/BDMA system 
initiated cure process $\left(T_{\max 2}=185^{\circ} \mathrm{C}\right)$. It confirmed that the second maximum was directly connected with the reaction between epoxy and amine, resulting in ether linkage formation. This reaction was probably catalyzed by the presence of hydroxyl groups in polyester's structure due to the formation of hydrogen-bonding groups [11, 12]. The total heat of polymerization $\left(\Delta H_{1}+\Delta H_{2}\right)$ associated with the peak for BPO/BDMA initiated cure reaction was lower than the sum of the heat for only BPO and only BDMA $\left(\Delta H=230 \mathrm{Jg}^{-1}\right)$ initiated. It could be explained by chemical interactions between BPO and BDMA components. BDMA catalyzed BPO decomposition to radicals, which initiated the cure process. In addition, during this decomposition, some amount of amine was transformed to the non-reactive tertiary amine cation in the epoxy-amine curing reaction, which probably caused the reduction in the total heat of polymerization. The presence of one signal with two maxima suggested that the curing mechanism of each component: carbon-carbon double bonds and epoxy groups were affected by presence of the other. So it was not possible to separate the radical polymerization from the epoxy-amine reaction for used curing system. Both processes run simultaneously and thus the increase of crosslinking density of the matrix with progressing curing caused increased $T_{\text {end }}$ values for BPO/BDMA initiating system compared to those only initiated by BPO. The movement of reacting groups due to the steric hindrance was reduced so decreasing the rate of the cure process was expected.

However, the cure process initiated by BPO/DMP-30 system followed a different course by comparison to BPO/ BDMA initiated. The DSC data are summarized in Tables 5 and 6. DSC curves for these samples are shown in Figs. 5 and 6. The two effects are clearly visible in the

Table 5 DSC data of the cure reaction of unsaturated epoxy polyesters based on EG, BDO, or HDO: different styrene content (20-80 wt $\%$ ) initiated by BPO $(1.0 \mathrm{wt} \%) / \mathrm{DMP}-30$

\begin{tabular}{|c|c|c|c|c|c|c|c|}
\hline Polyester & Styrene content/wt $\%$ & $T_{\max 1} /{ }^{\circ} \mathrm{C}$ & $T_{\max 2} /{ }^{\circ} \mathrm{C}$ & $T_{\text {end }} /{ }^{\circ} \mathrm{C}$ & $\Delta H_{1} / \mathrm{J} \mathrm{g}^{-1}$ & $\Delta H_{2} / \mathrm{J} \mathrm{g}^{-1}$ & $T_{\mathrm{d}} /{ }^{\circ} \mathrm{C}$ \\
\hline \multirow[t]{4}{*}{ EG } & 20 & 119 & 238 & 312 & 25 & 210 & 390 \\
\hline & 40 & 140 & 240 & 310 & 48 & 215 & 395 \\
\hline & 60 & 145 & 240 & 310 & 65 & 217 & 405 \\
\hline & 80 & 152 & 244 & 296 & 79 & 215 & 415 \\
\hline \multirow[t]{4}{*}{$\mathrm{BDO}$} & 20 & 118 & 240 & 312 & 23 & 208 & 398 \\
\hline & 40 & 141 & 242 & 310 & 46 & 214 & 405 \\
\hline & 60 & 143 & 242 & 308 & 67 & 210 & 410 \\
\hline & 80 & 152 & 243 & 300 & 78 & 212 & 415 \\
\hline \multirow[t]{4}{*}{ HDO } & 20 & 120 & 241 & 307 & 23 & 210 & 409 \\
\hline & 40 & 140 & 245 & 309 & 44 & 215 & 410 \\
\hline & 60 & 143 & 245 & 312 & 62 & 215 & 415 \\
\hline & 80 & 155 & 244 & 298 & 75 & 210 & 420 \\
\hline
\end{tabular}

Table 6 DSC data of the cure reaction of unsaturated epoxy polyesters based on EG, BDO, or HDO: styrene (20 wt $\%)$ initiated by different BPO concentration (0.5-3.0 wt \%)/DMP-30

\begin{tabular}{|c|c|c|c|c|c|c|c|}
\hline Polyester & BPO concentration $/ \mathrm{wt} \%$ & $T_{\max 1} /{ }^{\circ} \mathrm{C}$ & $T_{\max 2} /{ }^{\circ} \mathrm{C}$ & $T_{\text {end }} /{ }^{\circ} \mathrm{C}$ & $\Delta H_{1} / \mathrm{J} \mathrm{g}^{-1}$ & $\Delta H_{2} / \mathrm{J} \mathrm{g}^{-1}$ & $T_{\mathrm{d}} /{ }^{\circ} \mathrm{C}$ \\
\hline \multirow[t]{4}{*}{ EG } & 0.5 & 119 & 240 & 312 & 23 & 225 & 395 \\
\hline & 1.0 & 119 & 238 & 312 & 25 & 210 & 390 \\
\hline & 2.0 & 120 & 238 & 310 & 25 & 202 & 394 \\
\hline & 3.0 & 121 & 241 & 312 & 24 & 194 & 391 \\
\hline \multirow[t]{4}{*}{$\mathrm{BDO}$} & 0.5 & 120 & 242 & 312 & 24 & 220 & 395 \\
\hline & 1.0 & 118 & 240 & 312 & 23 & 208 & 398 \\
\hline & 2.0 & 121 & 240 & 310 & 27 & 202 & 399 \\
\hline & 3.0 & 119 & 240 & 309 & 28 & 192 & 395 \\
\hline \multirow[t]{4}{*}{ HDO } & 0.5 & 120 & 243 & 308 & 25 & 225 & 405 \\
\hline & 1.0 & 120 & 241 & 307 & 23 & 210 & 409 \\
\hline & 2.0 & 119 & 241 & 309 & 25 & 200 & 410 \\
\hline & 3.0 & 118 & 239 & 310 & 27 & 195 & 406 \\
\hline
\end{tabular}




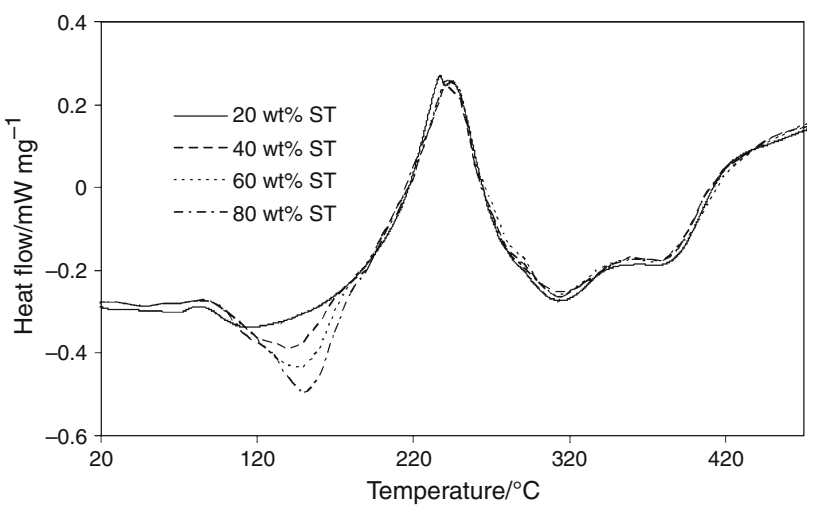

Fig. 5 DSC curves of the cure reaction of unsaturated epoxy polyester based on BDO-different styrene content (20-80 wt\% ST) initiated by $1.0 \mathrm{wt} \%$ BPO/DMP-30 system

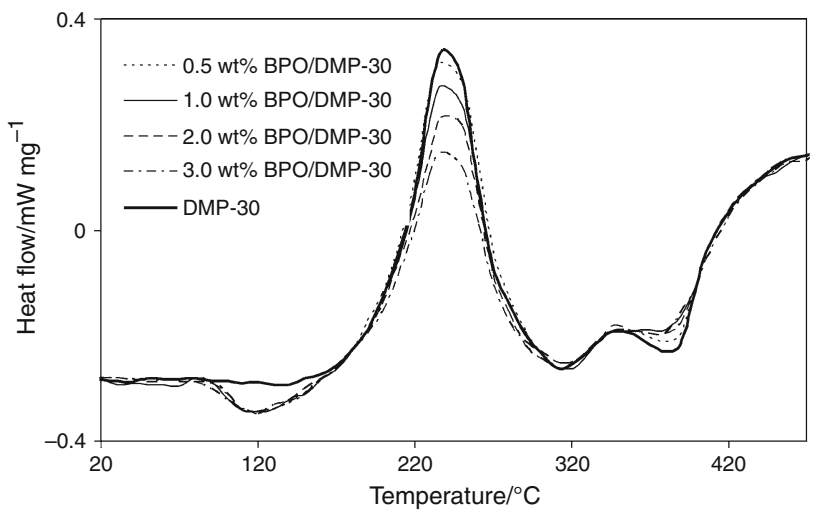

Fig. 6 DSC curves of the cure reaction of unsaturated epoxy polyester based on BDO-styrene (20 wt $\%$ ST) initiated by different BPO concentration/DMP-30 system

temperature range from about $80{ }^{\circ} \mathrm{C}$ to about $320{ }^{\circ} \mathrm{C}$. The first endothermal signal $\left(T_{\max 1}\right)$ was probably connected with the evaporation of un-reacted styrene. This observation was confirmed by $\Delta H_{1}$ values. The $\Delta H_{1}$ values increased with increasing styrene content in the studied compositions, Table 5. The position of the second exothermal peak $\left(T_{\max 2}\right)$ was independent of styrene content and BPO concentration. Moreover, $T_{\max 2}$ values for BPO/ DMP-30 initiated cure were comparable to those observed for only DMP-30 $\left(T_{\max 2}=238{ }^{\circ} \mathrm{C}\right)$ initiated where only polyether linkage formation was expected. The $\Delta H_{2}$ values of BPO/DMP-30 initiated cure reaction were a bit lower than those obtained when only DMP-30 was used as a hardener $\left(\Delta H_{2}=250 \mathrm{Jg}^{-1}\right)$. Also, $\Delta H_{2}$ values were almost independent of styrene content in the compositions and directly depended on BPO concentration. The $\Delta H_{2}$ values decreased with increasing BPO concentration, Table 6. All those observations suggested that the redox reaction between BPO and DMP-30 may result in non-radical products or radicals incapable of initiating the polymerization process. This behavior probably was directly connected with the presence of hydroxyl group in DMP-30 structure or due to the steric hindrance. First, the transfer of BPO radicals to hydroxyl group of DMP-30 through hydrogen abstraction from the tertiary amine was expected. During this transfer process there was a possibility of generation of a radical from the tertiary amine, which was incapable of initiating the polymerization reaction. Thus, some amounts of the amine might be consumed which resulted in a decrease in the overall rate of polymerization or causing complete inhibition of the polymerization process of carbon-carbon double bonds of polyesters and styrene $[4,25]$. Second, based on literature data, the presence of electron releasing substituents on the aromatic ring, facilitated the nucleophilic attack of nitrogen on peroxide oxygen, resulting in faster initiation of the cure process. However, this process was more pronounced when the electron releasing group is para or meta than ortho to the amine group [9]. Due to this, the branched DMP-30 structure and the presence of electron releasing group in ortho to two of three amine groups could act as a steric hindrance causing the amine to be a very sluggish promoter. In this case, only the exothermic peak connected with the formation of polyether linkages during the reaction between epoxy groups and amine groups was clearly observed.

\section{Conclusions}

The influence of chosen tertiary aromatic amines: BDMA or DMP-30 as potential promoters of the cure reaction of the unsaturated epoxy polyester resins containing different styrene content $(20-80 \mathrm{wt} \%)$ initiated by various $\mathrm{BPO}$ concentration $(0.5-3.0 \mathrm{wt} \%)$ has been studied by nonisothermal DSC. It was proved that the structure of the tertiary aromatic amine used significantly influenced on the course of the cure process. BPO/BDMA initiated cure reaction of the resins in this study started at relatively lower $T_{\text {onset }}$ temperatures compared to those only initiated by BPO. Also, the total heat of polymerization $\left(\Delta H_{1}+\Delta H_{2}\right)$ associated with the peak for BPO/BDMA initiated cure reaction was lower than the sum of the heat for only BPO and only BDMA initiated. This directly confirmed the chemical interactions between BPO and BDMA components. The organic peroxide-amine interactions caused the promotion of BPO decomposition to benzoyloxy radicals and thus accelerated the copolymerization process (lower $T_{\text {onset }}$ values). In addition, due to this process some amount of the amine was transformed to the non-reactive tertiary amine cation in the epoxy-amine curing reaction, which consequently caused the reduction in the total heat of polymerization. Moreover, the epoxy peak $\left(T_{\max 2}\right)$ was visible at similar temperatures for BPO/BDMA and only 
BDMA initiated cure process. This confirmed the occurring epoxy-amine reaction, probably catalyzed by the presence of hydroxyl groups in polyester's structure. The presence of a single broad calorimetric signal for BPO/BDMA initiated cure process suggests that the radical polymerization and the epoxy-amine curing reaction run simultaneously, so for used curing system, the heating rate should help to separate the processes, assuming that they have different activation energies.

However, the results obtained for BPO/DMP-30 initiated cure reaction of the studied resins were quite surprising. BPO/DMP-30 initiated cure process of the polyesters studied with styrene was quite different course comparing to those BPO/BDMA initiated. Based on the data reported, it was found, that DMP-30 was not suitable as a promoter for $\mathrm{BPO}$ decomposition, probably due to the presence of hydroxyl groups in their structure and/or the steric hindrance (their more branched structure). The possible transfer of BPO radicals to hydroxyl group of DMP30 through hydrogen abstraction from the tertiary amine used resulted in non-radical products or radicals incapable of initiating the polymerization reaction. Thus, some amount of DMP-30 was consumed which resulted in decrease in the overall rate of polymerization or causing complete inhibition of polymerization process of carboncarbon double bonds of polyesters and styrene. The calorimetric signal describing the evaporation of styrene was clearly indicated. In addition, the branched DMP-30 structure and the presence of electron releasing group in ortho to two of three amine groups could act as a steric hindrance causing the amine as a very sluggish promoter in the redox reactions. In this case, only the epoxy-amine cure reaction occurred, suggesting the formation of polyether linkages.

Open Access This article is distributed under the terms of the Creative Commons Attribution Noncommercial License which permits any noncommercial use, distribution, and reproduction in any medium, provided the original author(s) and source are credited.

\section{References}

1. Putatcioglu SY, Fricke H, Hasser JC. Heats of reaction and kinetics of a thermoset polyester. J Appl Polym Sci. 1979;24: 937-46.

2. Calvo S, Escribano J, Prolongo MG, Masegosa RM, Salom C. Thermomechanical properties of cured isophtalic polyester modified with poly(epsilon-caprolactone). J Therm Anal Calorim. 2011;103:195-203.
3. Yang H, Lee LJ. Comparison of unsaturated polyester and vinylester resins in low temperature polymerization. J Appl Polym Sci. 2000;79:1230-42.

4. Sarac AS. Redox polymerization. Prog Polym Sci. 1999;24: 1149-204.

5. Lin CP, Tseng JM, Chang YM, Liu SH, Cheng YC, Shu CM. Modeling liquid thermal explosion reactor containing tert-butyl peroxybenzoate. J Therm Anal Calorim. 2010;102:587-95.

6. Gao JG, Zhang X, Huo L, Zhao H. Curing reaction of o-cresolformaldehyde epoxy/LC epoxy (p-PEPB)/anhydride (MeTHPA). J Therm Anal Calorim. 2010;100:225-32.

7. Gonzalez MG, Cabanelas JC, Pozuelo J, Baselga J. Preparation of cycloaliphatic epoxy hybrids with non-conventional amine curing agents. J Therm Anal Calorim. 2011;103:717-23.

8. Wang HM, Zhang YC, Zhu LR, Zhang BL, Zhang YY. Synthesis and curing behavior of a novel liquid crystalline epoxy resins. J Therm Anal Calorim. 2011;103:1031-7.

9. Storey RF, Sudhakar D, Hogue M. Tertiary aromatic amines as cure reaction promoters for unsaturated polyester resins. II. Low temperature studies. J Appl Polym Sci. 1986;32:4919-30.

10. Brojer Z, Hertz Z, Penczek P. Żywice Epoksydowe.Warszawa: WNT; 1982.

11. Horie K, Hiura H, Sawada M, Mita I, Kambe H. Calorimetric investigation of polymerization reactions. III. Curing reaction of epoxides with amines. J Polym Sci A. 1970;8:1357-72.

12. Smith IT. The mechanism of the crosslinking of epoxide resins by amines. Polymer. 1961;2:95-108.

13. Boenig H. Unsaturated polyesters: structure and properties. Amsterdam: Elsevier; 1964.

14. Parkin B, Lamb F, Clifton BV. Polyesters. Vol. 2, Chap. 5. London: Iiiffe Books; 1967. p. 56-67.

15. Pastorino RL. In: Bruins PF, editor. Unsaturated polyester technology. New York: Gordon and Breach; 1967. p. 63-84.

16. Sheppard CS, Kamath VR. The selection and use of free radical initiators. Polym Eng Sci. 1979;19:597-606.

17. Worzakowska M. Chemical modification of unsaturated polyesters. Influence of polyester's structure on thermal and viscoelastic properties of low styrene content copolymers. J Appl Polym Sci. 2009;114:720-31.

18. Worzakowska M. Curing reaction of unsaturated (epoxy) polyesters based on different aliphatic glycols. J Therm Anal Calorim. 2010;102:745-50.

19. Królikowski W, Kłosowska-Wołkowicz Z, Penczek P. Żywice i laminaty poliestrowe. Warsaw: WNT; 1986.

20. Cao X, Lee LJ. Control of shrinkage and residual styrene of unsaturated polyester resins cured at low temperatures. I. Effect of curing agent. Polymer. 2003;44:1893-902.

21. Scott FT, Cook WD, Forsythe JS. Kinetics and network structure of thermally cured vinyl ester resins. Eur Polym J. 2002;38:705-16.

22. Tong LKJ, Kenyon WO. Heats of polymerization. III. Styrene and substituted styrenes. J Am Chem Soc. 1947;69:1402-5.

23. Flory PJ. Principles of polymer chemistry. Ithaca: Cornell University Press; 1953.

24. Severini F, Gallo R. Differential scanning calorimetry study of thermal decomposition of benzoyl peroxide and 2, 2'-azobisisobutyronitrile mixtures. J Therm Anal. 1985;30:841-7.

25. Dean K, Cook WD, Zipper MD, Burchill P. Curing behavior of IPNs formed from model VERs and epoxy systems I amine cured epoxy. Polymer. 2001;42:1345-59. 